\title{
Diagnosis of Human Toxoplasmosis Using Rapid Chromatographic Immunoassay and Enzyme-Linked Immuno-Sorbent Assay (ELISA) Compared to Molecular Technique (PCR) as Gold Standard Technique
}

\author{
Kareem Abdal Razaq Mouhamed $^{1 *}$, Abdel-Kareem A. AL-Kazzaz ${ }^{2}$ and Atif S.M. Idrees ${ }^{3}$ \\ ${ }^{1}$ College of Graduate Studies, Al-Neelain University Sudan \\ ${ }^{2}$ Department of Biotechnology, College of Science, University of Baghdad, Iraq \\ ${ }^{3}$ Department of Biology and Biotechnology, Faculty of Science and Technology, \\ Al-Neelain University, Sudan \\ *Corresponding author
}

\begin{tabular}{|l|}
\hline Ke y w or d s \\
Toxoplasmosis, $T$. \\
gondii, Diagnosis, \\
Immunoblottest, \\
ELISA, PCR.
\end{tabular}

A B S T R A C T

Nowadays, there are many different procedures for the laboratory diagnosis of Toxoplasma infection in pregnant women, congenitally-infected fetuses and newborns, and they are mainly performed by serological testing, and PCR (for confirmatory purpose), beside many commercial diagnostic tests, which use Toxoplasma lysate antigen. These procedures differ from each other in many aspects; cost, time-consumption, and accuracy of the test, which should meet patient's needs. In this current study, serum samples were collected from one-hundred females suspected with toxoplasmosis and diagnosed by three different procedures for the $T$. gondii infection, rapid chromatographic (IgM or IgG immunoblot), ELISA (IgM or IgG) test and molecular technique. Molecular diagnoses were performed in peripheral blood by PCR using the $T$. gondiiB1 gene as marker. The results were described as frequency and percentage of positivity; also, specificity and sensitivity were assessed. Of these 100 blood samples analyzed, 92\% (IgG), and 55\% (IgM) were positive when using PCR; the rapid chromatographic method for both IgM and IgG, has shown (83\%) samples to be positive for T. gondii, while in ELISA test, $28 \%$ $(\operatorname{IgM})$ and $72 \%(\operatorname{IgG})$ found to be positive. Sensitivity and specificity of ELISA (89\% and $90 \%$ (IgM); $89 \%$ and $91 \%$ (IgG), respectively) have found to be relatively higher than immunoblot (88\%and $81 \%(\mathrm{IgM}) ; 85 \%$ and $81 \%(\mathrm{IgG})$, respectively). While PCR technique has shown $100 \%$ and $98.8 \%(\operatorname{IgM}) ; 99.3 \%$ and $96.1 \%(\operatorname{IgG})$, sensitivity and specificity, respectively. Since the higher sensitivity and specificity of ELISA, we concluded that ELISA, compared to the rapid chromatographic test, is more suitable for the detection of anti-T. gondii IgG and IgM antibodies in both acute and chronic infection, especially, the rapid chromatographic commercial kits can yield many false-positive results which in turn has many undesired consequences.

\section{Introduction}

Despite the advance techniques in diagnosis of parasitic disease, and protozoan disease; diagnostic methods must be renewed to be more rapid and specific. During the past few years, there has been an increased interest in the diagnosis of parasitic diseases using 
techniques, which are rapid, simple and inexpensive as well as sensitive and specific (Dubey et al., 2005). Old serological procedures such as indirect hem agglutination (Dubey and Su, 2009), complement fixation test (Linguissi, 2012) and immunofluorescence are tedious and difficult to standardize, conduct and interpret. Also, the reagents are consumptive and require highly trained technicians as well as expensive instruments (Joanna et al., 2009). T. gondii is a coccidian parasite of the cat and its infection may lead to major public health problems (Evering and Weiss, 2006). The disease exhibits various clinical manifestations and therefore, poses difficulty in diagnosis (Rai et al., 1995). Serological methods have been employed in aid of diagnosis of this disease. Detection of anti-toxoplasma $\operatorname{IgG}$ and $\operatorname{IgM}$ antibodies have been routinely used in many clinical laboratories to determine the probable immune status of individuals. The most used assay today is indirect enzyme linked immunosorbent assay (ELISA); require highly trained and expensive instruments (Sroka et al., 2010). So, this research was planned, in parallel with rapid latex agglutination test and enzyme linked immunosorbent assay (ELISA), to standardize the commercially available Rapid chromatographic immunoassay (immunoblotting) technique, which is simple to perform and doesn't need expensive equipment to detect $\operatorname{IgG}$ and $\operatorname{IgM}$ specific antibodies against Toxoplasma gondii.

\section{Materials and Methods}

\section{Samples}

One hundred peripheral blood samples were collected, at the first visit, from females who presented at Obstetrics and Gynecology Department, at AL-Yarmoke Hospital and private outpatient clinics in Baghdad, Iraq, during September 2016- September 2017, and their clinical details at presentation were recorded. Sera were stored at $-20{ }^{\circ} \mathrm{C}$ till analyzed.

\section{Polymerase chain reaction}

DNA was extracted from patients' blood using DNeas Kit (Qiagen, Hilden, Germany). The Toxoplasma gondiiBl gene (sequence of 592 bp) was amplified as described previously (8). Primer pair used had the nucleotide sequence as follows:

Forward primer: GCATTCCCGTCCAAACT

\section{Reverse primer: AGACTGTACGGAAT GGAGACGAA}

The PCR conditions consisted of 1 cycle of 5 min at $93^{\circ} \mathrm{C}$, followed by 35 cycles of $1 \mathrm{~min}$ at $93^{\circ} \mathrm{C}, 30 \mathrm{~s}$ at $55^{\circ} \mathrm{C}, 30 \mathrm{~s}$ at $72^{\circ} \mathrm{C}$, and a final cycle of $10 \mathrm{~min}$ at $72^{\circ} \mathrm{C}$. Amplified products were visualized on $2 \%$ agarose gel under UV light. All assays were performed at least twice.

\section{Rapid chromatography test}

This test utilizing anti-T. gondii $\operatorname{IgG}$ and $\operatorname{IgM}$ antibodies rapid test, which is a qualitative, chromatographic immunoassay for detection of IgM, IgG antibodies against $T$. gondii antigen in patient's sera. It was performed according to the evolved instructions.

The procedure assay can be summary as follow: sample, controls and calibrator were diluted 1:40 by adding $5 \mu / 200 \mu 1$. Reaction was stoppedby $100 \mu \mathrm{l}$ stop solution and read at $450 \mathrm{~nm}$. All results above the cut-off value (10 IU/Ml) were considered as positive.

\section{Enzyme Linked Immunosorbent Assay (ELISA)}

The Biocheck® Kit was commercial obtained. Diluted patient's serum was added to the purified $T$. gondii antigen coated on the 
surface of micro wells. The T. gondii $\mathrm{IgG}$ or IgM-specific antibody, if present, binds to its antigen, all unbound materials were washed away, then horse-radish peroxidase (HRP) conjugate was added, which binds to the antibody-antigen complex. After washing, the solution of tetra-methyl benzidine (TMB) reagent was added, the enzyme conjugate catalytic was stopped at specific time. The results were read by ELISA reader.

\section{Statistical analysis}

All data were presented as frequency and percentage of positive and negative results, and the cutoff value was determined. Sensitivity and specificity were calculated for each procedure as the following equations:

Sensitivity $=a /(a+c)$

Specificity $=d /(b+d)$

Whereas;

$a=$ True positive

$b=$ False positive

$c=$ False negative

$d=$ True negative

\section{Results and Discussion}

In the present study, 100patients suspected with $T$. gondii samples were tested between 2016 and 2017. Of these 100 blood samples analyzed, 92\% (IgG) and 55\% (IgM) were positive when using PCR; the rapid chromatographic method for both $\operatorname{IgM}$ and $\mathrm{IgG}$, has shown $83 \%$ of samples to be positive for $T$. gondii, while in ELISA test, $72 \%$ of samples were $\operatorname{IgG}$ positive and $28 \%$ samples were IgM positive (Figure 1).

The evaluation of results obtained has shown that PCR test has $100 \%$ and $98.8 \%(\operatorname{IgM})$; $99.3 \%$ and $96.1 \%$ (IgG), sensitivity and specificity, respectively, ELISA has shown
$89 \%$ and $90 \%(\operatorname{IgM}) ; 89 \%$ and $91 \%(\operatorname{IgG})$, respectively, whereas in rapid chromatographic test it has shown $88 \%$ and $81 \%(\operatorname{IgM}) ; 85 \%$ and $81 \%$ (IgG), respectively (Table 1).

In developed countries, toxoplasmosis screening is part of the health tests included in the prenatal assessment. Toxoplasmosis contracted during the first trimester of pregnancy is responsible for spontaneous abortions, stillbirth or severe illness in more than $25 \%$ of pregnant women (Su et al., 2010).

The aim of the current study was to determine the sero-prevalence of toxoplasmosis among pregnant women during the first trimester of pregnancy, in addition, to compare the common diagnosis tests in comparison to PCR technique.

In this study, of 100 sera of pregnant women, 83 samples (83\%) showed positive for $T$. gondii by rapid chromatographic test for both IgM and IgG, while in ELISA test, 28\% and $72 \%$ samples were IgM and IgG positive, respectively. The PCR technique has shown 92\% (IgG) and 55\% (IgM) positive cases. All cases have shown positivity for both $\operatorname{IgM}$ and IgG antibodies against $T$. gondii antigens.

A positive Toxoplasma Immunoglobulin $\mathrm{M}$ (IgM) result is regularly interpreted as an indicator of an acute infection. Though, IgM can persist for many years; however, Toxoplasma commercial IgM diagnostic test kits can yield a number of false-positive results. For these reasons, a chronic Toxoplasma infection can be erroneously classified as an acute infection, resulting in serious adverse consequences, especially in pregnant women, leading to emotional distress and unnecessary interventions, including termination of pregnancy (Dhakal et al., 2015). 
Fig.1 T. gondii cases diagnosed by PCR, ELISA, Chromatography

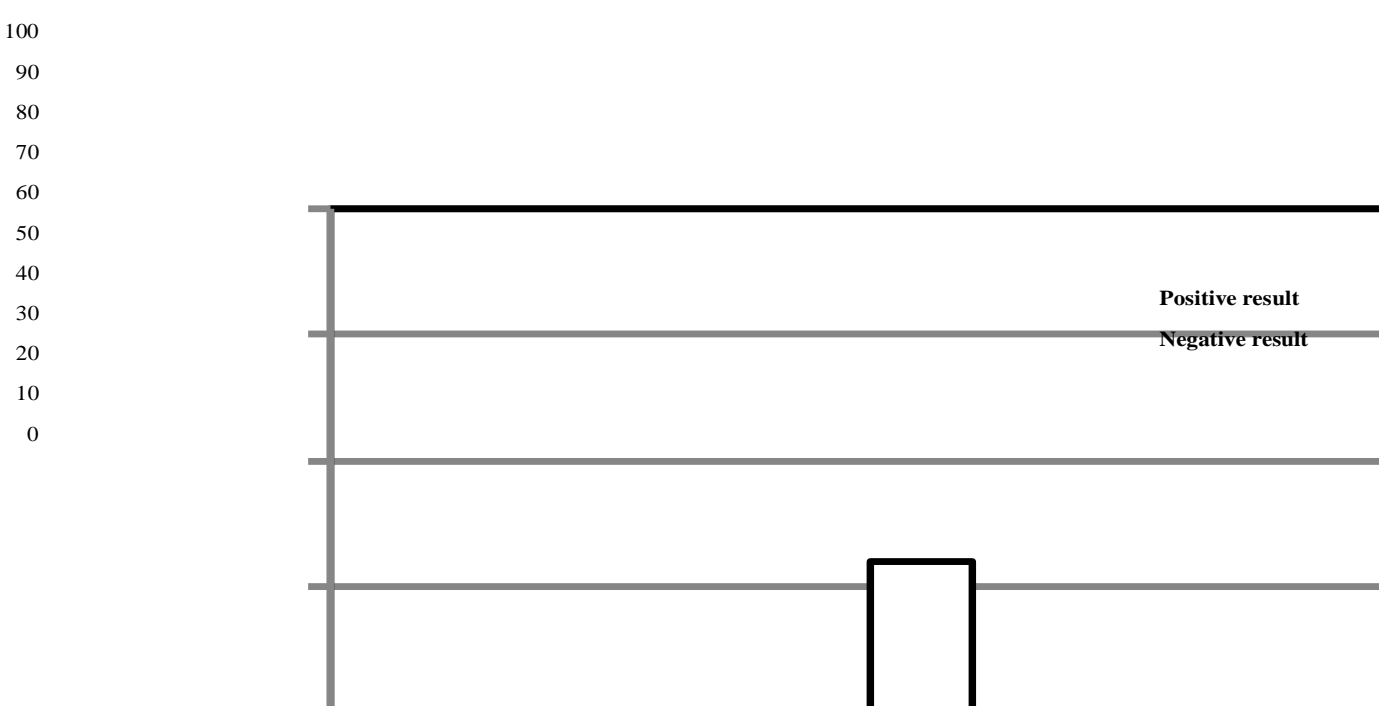

Table.1 Results of specificity and sensitivity ELISA of and Rapid test (IgG and IgM) according to PCR results

\begin{tabular}{|l|c|c|c|c|c|c|} 
& \multicolumn{2}{|c|}{ PCR } & \multicolumn{2}{c|}{ ELISA } & \multicolumn{2}{c|}{ Chromatography } \\
\hline Specificity & IgG & IgM & IgM & IgG & IgG & IgM \\
\hline Sensitivity & $99.3 \%$ & $100 \%$ & $90 \%$ & $91 \%$ & $86 \%$ & $81 \%$ \\
\hline
\end{tabular}

On the other hand, few women seek toxoplasmosis serologies during pregnancy in Baghdad; this could be due to the relatively high cost of these tests, especially, for predominantly low-income populations. In Baghdad, and due to the existence of a strong agro-pastoral activity especially in rural areas, which increases the spread of zoonotic diseases, Toxoplasmosis diagnosis should be systematic (Coulibaly and Yameogo, 2000). Indeed, previous studies have shown that the coexistence between humans and animals may be a contributing factor raising these zoonotic infections (Sroka et al., 2010; Su et al., 2010; Coulibaly and Yameogo, 2000; Rovamycine, 1994). Meat and milk are important dietary components for most of the population in Baghdad. However, contamination of water by oocytes could be the most likely source of infection with toxoplasmosis in Baghdad. The diagnosis of toxoplasmosis is necessary in pregnant women because of the low immunization coverage rate and the high level of exposure to these two infections which can be harmful to the newborn if contracted by women before the third trimester of pregnancy (Coulibaly and Yameogo, 2000).

Since the higher sensitivity and specificity of ELISA, we concluded that ELISA, compared to the rapid chromatographic test, is more suitable for the detection of anti-T. gondii IgG and $\operatorname{IgM}$ antibodies in both acute and chronic infection, especially, the rapid chromatographic commercial kits can yield many false-positive results which in turn has many undesired consequences. 


\section{Supplements}

\section{Instruction for the rapid chromatography test}

\section{Negative control}

Only the control band (C band) shows color development. The two test bands (T1 and T2) show no color development.

\section{Positive control}

The $\mathrm{C}$ band and two $\mathrm{T}$ bands (T1and $\mathrm{T} 2$ ) show color development.

\section{Interpretation of assay results; Negative} result

If only the $\mathrm{C}$ band is present, the absence of color in both $\mathrm{T}$ bands (T1 and T2) indicates that no anti- $T$. gondii antibodies are detected (result is negative);

\section{Positive result}

In addition to the presence of Cband, if only $\mathrm{T} 1$ band color is developed indicate the IgM anti-Toxoplasma is presence in the specimen (IgM positive). While if only $\mathrm{T} 2$ band is developed indicate the (IgG positive) and if both $\mathrm{T} 1$ and $\mathrm{T} 2$ bands are developed in addition to the presence of $\mathrm{Cb}$ and that means both (IgM and IgG is positive), if noC band is developed, the assay is invalid regardless of any color in the $\mathrm{T}$ bands.

\section{References}

Coulibaly ND, and Yameogo KR, 2000. Prevalence and control of zoonotic diseases: collaboration between public health workers and veterinarians in Burkina Faso. Acta Trop 76(1): 53-57.

Dhakal R, Gajurel K, Pomares C, Talucod J, Press CJ, Montoya JG. 2015.
Significance of a positive Toxoplasma Immunoglobulin $\mathrm{M}$ test result in the United States. J Clin Microbiol 53:3601-3605. doi:10.1128/JCM.01663-15.

Dubey JP, and Su C, 2009. Population biology of Toxoplasma gondii: what's out and where did they come from. Journal of MemInst Oswaldo Cruz, 104 (2) $190-1951$.

Dubey JP, Hill DE, Jones JL, 2005. Prevalence of viable Toxoplasma gondii in beef, chicken, and pork from retail meat stores in the United States: risk assessment to consumers. J Parasitol, (91)1082-93.

Evering, T. and Weiss, L.M, 2006. The immunology of parasite infections in immune compromised hosts. Parasite Immunology, (28) 549-565.

Joanna M., Violetta D., Halina K, 2009. Does Toxoplasma gondii Infection affect the Levels of IgE and Cytokines (IL-5, IL6, IL-10, IL-12, and TNF-alpha)? Clin Dev Immunol. (15) 374-396.

Linguissi L, 2012. Seroprevalence of toxoplasmosis and rubella in pregnant women attending antenatal private clinic at Ouagadougou, Burkina Faso. Asian Pac J. Trop Med, 5(10):810-813.

Rai R., Clifford K., Cohen H., Regan L, 1995. High prospective fetal loss rate in untreated pregnancies of women with recurrent miscarriage and antiphospholipid antibodies. Hum. Reprod, (10)3301-3304.

Rovamycine (Rhone-Poulenc Rorer), 1994. In: Vidal. 70th ed. Paris: Editions du Vidal, 1361.

Sroka J, Wojcik-Fatla A, Szymanska J, Dutkiewicz J, Zajac V, Zwolinski J, 2010. The occurrence of Toxoplasma gondii infection in people and animals from rural environment of Lublin region - estimate of potential role of water as a source of infection. Ann Agric Environ 
Med 17(1): 125-132.

Su C, Shwab EK, Zhou P, Zhu XQ, Dubey

JP, 2010. Moving towards an integrated approach to molecular detection and identification of Toxoplasma gondii. Parasitol J, 137(1) 1-11.

\section{How to cite this article:}

Kareem Abdal Razaq Mouhamed, Abdel-Kareem A. AL-Kazzaz and Atif S.M. Idrees. 2018. Diagnosis of Human Toxoplasmosis Using Rapid Chromatographic Immunoassay and Enzyme-Linked Immuno-Sorbent Assay (ELISA) Compared to Molecular Technique (PCR) as Gold Standard Technique. Int.J.Curr.Microbiol.App.Sci. 7(01): 3150-3155.

doi: https://doi.org/10.20546/ijcmas.2018.701.373 